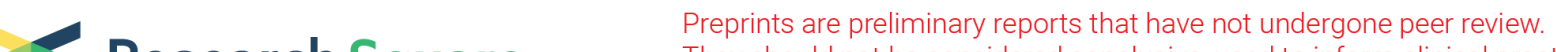 Research Square They should not be considered conclusive, used to inform clinical practice, or referenced by the media as validated information.
}

\section{Anti-Tumor Activity and Mechanism of Gemcitabine Derivative SZY-200 Assembled from Gemcitabine- Lauric Acid Conjugate on Human Bladder Cancer Cells}

\section{Hongxia Wang}

East China University of Science and Technology

\section{Zhiyu Shao}

Donghua University College of Materials Science and Engineering

\section{Zhiwen Xu}

East China University of Science and Technology

\section{Binghao Ye}

East China University of Science and Technology

\section{Qiaoqiao Zheng}

East China University of Science and Technology

\section{Ming Li}

East China University of Science and Technology

\section{Xingyuan $\mathrm{Ma}$}

East China University of Science and Technology

\section{Ping Shi ( $\nabla$ ship@ecust.edu.cn )}

East-China Institute of Technology: East China University of Science and Technology https://orcid.org/0000-0002-6904-2802

\section{Research Article}

Keywords: SZY-200, Bladder cancer, hENT1, Cell cycle arrest, Apoptosis

Posted Date: September 8th, 2021

DOl: https://doi.org/10.21203/rs.3.rs-867327/v1

License: (c) (1) This work is licensed under a Creative Commons Attribution 4.0 International License. Read Full License 


\section{Abstract}

Gemcitabine is a first-line drug for the treatment of bladder cancer. One of the most important mechanisms of gemcitabine resistance is the low expression of cellular membrane transporter hENT1. Various derivatives containing fatty acid side chains have been developed in order to facilitate gemcitabine uptake and prolong its retention in cells, such as CP-4126. In this study, the anti-tumor effect and mechanism of a new derivative of gemcitabine named SZY-200 on bladder cancer cells was investigated. SZY-200 was assembled from gemcitabine-lauric acid conjugate. Our data showed that SZY-200 could inhibit the proliferation of bladder cancer cells by inducing cell cycle arrest and apoptosis. The inhibitory effects were comparable to gemcitabine and CP-4126. Importantly, SZY-200 was independent of the membrane transport system in bladder cancer cells. Moreover, we found that lauric acid could also inhibit the proliferation of bladder cancer cells. SZY-200 could downregulate the expressions of PPARG and PTGS2 which were related to the occurrence and development of bladder cancer. Overall, these results indicate that SZY-200 could be an ideal candidate drug for further in vivo investigation.

\section{Introduction}

Bladder cancer is the 10th most commonly diagnosed cancer worldwide, and approximately 573,000 new cases and 213,000 deaths in 2020 [1]. Gemcitabine (GEM) is currently the first-line option for treating bladder cancer and widely used for bladder perfusion therapy in patients with non-muscle invasive bladder cancer (NMIBC). Intravesical GEM seemed to be a valid and safe alternative at 3 years follow-up for patients who were unwilling to undergo radical cystectomy $(\mathrm{RC})$ or failed bacillus Calmette-Guérin (BCG) [2]. GEM is the most effective therapy considering both recurrence and progression outcomes [3]. In addition, the combination chemotherapy of GEM and cisplatin (GC) exhibited similar efficacy and less adverse events for patients with MIBC than the standard methotrexate plus vinblastine, doxorubicin and cisplatin (MVAC) therapy and high-dose MVAC $[4,5]$.

However, the drug resistance and toxicity severely limit utilization of GEM in clinics. GEM is a highly hydrophilic pyrimidine nucleoside analog. Human equilibrative nucleoside transporter 1 (hENT1) is the primary facilitative transporter of GEM [6]. High levels of hENT1 were correlated with high GEM concentration in cancer cells and increases drug efficacy $[7,8]$. Previous studies have indicated that high hENT1 expression was associated with longer overall survival (OS) among patients treated with both palliative and adjuvant GEM $[9,10]$ and vice versa $[11,12]$. The activation of GEM by deoxycytidine kinase (dCK) to form active diphosphate and triphosphate forms (dFdCDP and dFdCTP, respectively) is the rate-limiting step [13]. Furthermore, the majority ( 90\%) of intracellular GEM is inactivated by deamination by deoxycytidine deaminase (dCDA) to 2', 2'-difluorodeoxyuridine (dFdU) and subsequently degraded and excreted out of cells [14]. For another, oral administration of GEM was terminated because of high conversion of GEM and accumulation of $\mathrm{dFdU}$, which most likely resulted in severe liver toxicity [15]. Therefore, it is urgent to further modify and design novel GEM derivatives. 
CP-4126 is a GEM derivative assembled from GEM-elaidic acid (EA) conjugate, which has a broad spectrum anti-tumor activity in a wide range of human tumor models in vivo [16, 17]. Importantly, some studies have shown that CP-4126 is membrane transporter independent. CP-4126 is not the substrate for dCDA and can inhibit deamination of GEM [17-19]. However, EA, another hydrolyzed product of CP-4126 was reported to enhance the metastasis of colon cancer by increasing the stemness of cancer cells and epithelial-mesenchymal transition, and induce drug resistance [20]. In contrast, lauric acid (LA) is associated with certain health benefits of coconut oil intake and some studies highlighted its anti-tumor effects. LA contributes the least to fat accumulation among all fatty acids [21]. Soliman et al. [22] reported that a diet regimen of ketogenic breakfast along with supplementation with two doses of LA-rich medium-chain triglycerides at breakfast and lunch times could be a potential prophylactic strategy and adjuvant therapy to combat COVID-19 infections. LA could stimulate ketone body production in the KT-5 astrocyte cells, which improved brain health by providing fuel to neighboring neurons [23]. Moreover, LA has anti-proliferative and pro-apoptotic efficacy in colon, breast and endometrial cancer cells [24]. Therefore, Shao et al. designed a new active derivative of GEM by using LA to replace the EA in CP- 4126 and named SZY-200. The aim of the present study is to investigate the potential anti-tumor effect of SZY200 on bladder cancer cells and to further understand the underlying molecular mechanism in an effort to shed light on the potential development of SZY-200 in the treatment of bladder cancer, and even other types of cancers. Chemical structures of these drugs were shown in Fig. 1.

Fig. 1 Design strategies and chemical structures of GEM derivatives CP-4126 and SZY-200

\section{Results And Discussion}

\subsection{SZY-200 and LA suppress proliferation of bladder cancer cells}

Firstly, we evaluated the effects of SZY-200 and LA on the proliferation of various cells in vitro using CCK8 assay. GEM and CP-4126 were used as positive controls. We found that SZY-200 could significantly inhibit the proliferation of a variety of cancer cells in a dose-dependent manner and has a certain selectivity to the normal uroepithelium cell line SV-HUC-1 and human normal liver cell line QSG-7701 (Fig.2A and S1). Moreover, SZY-200, GEM and CP-4126 showed similar cytotoxic effects at the same dose and the human bladder cancer cells were more sensitive to them. While LA did not affect the proliferation of all tested cells at the same dose (Fig.2A and S1). We also compared the effects of LA with EA on the cell proliferation. The data showed that LA and EA had no significant growth inhibitory effects on UM-UC-3 and SV-HUC-1 cells (Fig.2A). However, the cell colony forming assay showed that LA could significantly inhibit the proliferation of UM-UC-3 cells in a dose-dependent manner. While EA had no inhibitory effect on the cells (Fig. 2B and 2C).

Figure 2 Antiproliferative activities of SZY-200 and LA against tumor cell lines with CP-4126, GEM and EA as positive control. (A) Cell viability after treatment for $48 \mathrm{~h}$. (B) Colony formation abilities of UM-UC-3 cells was determined after treatment with EA or LA. (C) Statistical analysis of (B). Values are expressed 
as the mean \pm SD from three independent experiments. ${ }^{* \star *} p<0.0001$ vs control. Abbreviations: GEM, GEM; EA, elaidic acid; LA, lauric acid

\subsection{SZY-200 is membrane transport system independent}

The differential expressions of nucleoside transport hENT1 mRNA in different cells were analyzed by qRTPCR. The data showed that the expression levels were basically consistent with the sensitivities of the cells to the three drugs (Fig. 3A, 2A and S1). Subsequently, in order to study the role of hENT1 for these three drugs, differences in sensitivity to the drugs in the presence of hENT1 inhibitor NBMPR were determined. NBMPR decreased sensitivity to GEM in bladder cancer cells, while sensitivities to SZY-200 and CP-4126 were increased (Fig. 3B-3D). Taken together, different from GEM, SZY-200 and CP-4126 were independent of the membrane transport system. That is, they do not rely on hENT1 to help them enter into bladder cancer cells.

Fig. 3 The expressions of hENT1 at the transcriptional level in different cells and the effects of the nucleoside transport inhibitor NBMPR on the cytotoxic activities of SZY-200, GEM and CP-4126 in bladder cancer cells. (A) The expression of hENT1 in different cells, $* \star \star * P<0.0001$ vs. the normal cells QSG-7701. Cell viability was measured at 48h after T24 cells (B), UM-UC-3 cells (C) and 5637 cells (D) were treated with SZY-200, GEM and CP-4126 (6.25, 12.5 and $25 \mathrm{nM})$ with or without $100 \mu \mathrm{M}$ NBMPR. Data were mean \pm S.D. $(n=3)$

\subsection{SZY-200 could arrest the cell cycle, induce apoptosis and significantly inhibit bladder cancer cells migration}

Flow cytometric analysis was performed to determine whether SZY-200 affected the cell cycle distribution in bladder cancer cells. As shown in Fig. 4A and 4B, similar to GEM and CP-4126, SZY-200 significantly induced G1-phase cell cycle arrest in UM-UC-3 cells. In T24 and 5637 cells, SZY-200 also was similar to GEM and CP-4126 and caused the cell cycle arrest at G2 phase (Fig. S2A and S2B).

Then, we quantified SZY-200-induced apoptosis of cells by flow cytometry. The percentage of total apoptotic cells was significantly increased in UM-UC-3 (Fig. 4C and 4D), T24 and 5637 (Fig. S2C and S2D) cells after treated by SZY-200, GEM and CP-4126. Meanwhile, Hoechst 33258 staining showed changes in cellular morphology in UM-UC-3, T24 and 5637 cells after treated by SZY-200, GEM and CP4126 (Fig. 4E, S2E). The untreated control cells were regular in shape, with abundant cytoplasm and also well attached to the membrane, the nuclei were round and stained homogeneously. On the contrary, the cells treated with three drugs were irregular in shape, the cell boundary was not smooth and the cells were more loosely attached to the membrane, there was lysis of some cells and showed evidence of blebbing. Moreover, apoptotic bladder cancer cells showed condensed and marked fragmented nuclei (white arrows) in a dose-dependent manner (Fig. 4E, S2E). Western blot analysis showed that the ratio of apoptosis-related proteins Bax and Bcl-2 were increased in UM-UC-3 cells after treated by SZY-200, CP4126 and GEM (Fig. 4F and 4G). The similar data were obtained in T24 cells except no change of the 
ratio of Bax and Bcl-2 after treated by GEM (Fig. S2F and S2G). Taken together, these data suggested that SZY-200 induced apoptosis of bladder cancer cells.

Subsequently, cell motility was assessed by light microscopy. As shown in Fig. $4 \mathrm{H}$ and $4 \mathrm{I}$, three drugs could significantly inhibit the migration of UM-UC-3 cells in a dose-dependent manner. The similar data were observed in both T24 and 5637 cells (Fig. S3A and S3B).

\section{Fig. 4 Effects of SZY-200 on cell cycle progression and the apoptosis rate in UM-UC-3 cells and scratch} tests. (A) After incubation, the cell cycle of UM-UC-3 cells was analyzed by flow cytometry. (B) Statistical analysis of the percentage of cells at the G0/G1, S and G2/M phases of cell cycle. (C) Apoptotic analysis was performed by flow cytometry using Annexin V-FITC/PI double staining method. (D) Statistical analysis of the percentages of apoptotic cells. (E) Apoptosis in UM-UC-3 cells was observed by fluorescence microscope using Hoechst 33258 staining (Scale bar $=50 \mu \mathrm{m}$ ). (F) Cell apoptosis-related protein expression in UM-UC-3 cells was detected by western blotting. (G) Represents the ratio of Bax/Bcl2 protein expression levels. ${ }^{* \star *} \mathrm{P} \otimes 0.0001$ vs control. $(\mathrm{H})$ Representative wound-healing assay pictures for UM-UC-3 cells treated with DMSO or with three drugs (12.5 and $25 \mathrm{nM}$ ) are shown (vertical lines indicate wound edges); (400x, original magnification). (I) Histograms represent quantitative analyses of cell

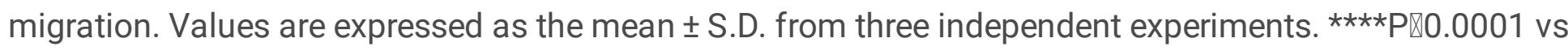
DMSO

\subsection{SZY-200 down-regulates the expression of peroxisome proliferator-activated receptor Y (PPARG), cyclooxygenase-2 (COX-2, also known as PTGS2) in bladder cancer cells}

Finally, BATMAN-TCM database was used to predict the target genes of GEM and LA. As shown in Fig. 5A, there are 47 target genes for GEM, 11 for LA and no intersection between them. Disease enrichment analysis from TTD database showed that among the target genes of LA, PPARG and PTGS2, were related to the occurrence and development of bladder cancer (as shown in the black box). Moreover, qRT-PCR analysis showed that the mRNA expression of PPARG and PTGS2 were upregulated in two bladder cancer cell lines in comparison to the normal cells SV-HUC-1 (Fig. 5B). Importantly, SZY-200, CP4126 and GEM could downregulate the expression of the two genes in bladder cancer cells (Fig. 5C).

Fig. 5 Prediction and analysis of target genes of GEM and LA. (A) Target genes from BATMAN-TCM database and disease enrichment analysis from TTD database. (B) Differential expressions of PPARG and PTGS2 in different cells. ${ }^{*} \mathrm{P} \unrhd 0.01,{ }^{*} * \star \mathrm{P} \otimes 0.0001$ vs SV-HUC-1. (C) Effects of three drugs on the expressions of PPARG and PTGS2 in bladder cancer cells. Values are expressed as the mean \pm SD from three independent experiments. ${ }^{* \star * * P 囚 0.0001 ~ v s ~ D M S O ~}$

Previous studies have shown that CP-4126 was well tolerated with comparable toxicity profile to GEM in patients with some advanced solid tumors [25]. In this study, SZY-200 and CP-4126 also showed similar cytotoxic effects in bladder cancer cells compared to GEM. The similar effect of SZY-200, CP-4126 and GEM demonstrated an effective conversion of the derivatives to GEM. CP-4126 was reported to traverse cell membranes by passive diffusion, followed by intracellular conversion to GEM by esterases in plasma 
and within tumor cells in order to be activated, and it is also dependent on dCK for phosphorylation [26]. In addition, CP-4126 mainly localized in the membrane and cytosolic fraction, which leads to the long retention inside the cell [19]. Compared with CP-4126, SZY-200 is assembled with LA not EA. Liu R et al. [27] once synthesized a drug (LA-Ara) based on the conjugation of cytarabine (Ara-C) with LA. The LA increased the lipophilicity of Ara-C and protected its $\mathrm{NH}_{2}$ group from the enzymatic attachment, thus markedly prolongs its plasma half-life. Furthermore, LA-Ara effectively improved anti-tumor activity compared with Ara-C. Importantly, LA-Ara could obviously decrease the incidence of toxic effects of Ara-C and is suitable to oral administration. From our data, clonogenic survival assay showed that LA could significantly inhibit the proliferation of bladder cancer cells in a dose-dependent manner, while EA has no inhibitory effect (Fig. 2B and 2C). Therefore, SZY-200 has the same or better anti-tumor activity as CP4126.

Several previous studies highlighted that a significant correlation between the levels of hENT1 expression and the $\mathrm{IC}_{50}$ values for GEM in cancer cells $[28,29]$. Neoptolemos et al. [30] demonstrated that patients with high hENT1 expression had a significantly higher median survival compared to patients with low hENT1 expression for those who received adjuvant GEM after undergoing surgical resection for pancreatic cancer. Galmarini et al. [18] found that the inhibition of hENT1 conferred resistance to GEM. In our study, the levels of hENT1 mRNA were found to basically be consistent with the sensitivities of the cells to GEM, CP-4126 and SZY-200. The hENT1 inhibitor NBMPR decreased sensitivity to GEM in bladder cancer cells and increased sensitivities to SZY-200 and CP-4126 (Fig. 3B-3D). Similarly, Bergman et al. (2011) reported that NBMPR and another nucleoside transport inhibitor dipyridamole decreased sensitivities to GEM 55 273-fold, while sensitivities to CP-4126 were only decreased 0 1.8-fold in THX human malignant melanoma cells and MOLT4 human T-cell leukemia cells. These studies revealed a nucleoside transporter independent transport over the cell membrane of CP-4126 and SZY-200. Moreover, hENT1 could operate as uptake as well as export transporter of GEM, the activity of SZY-200 and CP4126 could be improved by cellular accumulation and prolonged retention of GEM inside the cell because of the inactivation of hENT1 [19]. We therefore believe that SZY-200 could be more effective than GEM for bladder cancer patients with low hENT1 expression. For another, GEM was reported to inhibit the proliferation of bladder cancer cells by inducing cell cycle arrest and apoptosis [31, 32]. From our data, SZY-200 and CP-4126 exhibited the similar effects, which suggests that these GEM derivatives will release GEM in tumor cells to play its anticancer role. Interestingly, these drugs induced cell cycle arrest at G1-phase in UM-UC-3 cells, but G2-phase in T24 and 5637 cells (Fig. 4A, 4B, S2A and S2B). Several previous studies also showed similar results [33-35]. The underlying mechanism of this difference needs to be further explored in the future.

PPARG and PTGS2 are target genes of LA that we predicted by the BATMAN-TCM database. Previous studies have shown that they were related to the occurrence and development of bladder cancer. PPARG is a ligand-activated nuclear receptor and has been reported to interact with multiple signaling pathways, including Bcl-2, p53, p21, PTGS2 and cyclin D1 [36]. The reduction of the activity of PPARG, whether through drug inhibition or gene ablation, would inhibit the proliferation of bladder cancer cells [37-39]. 
PTGS2 is a prostaglandin endogenous peroxide synthase. Recent studies have shown that human bladder cancer is associated with increased expression of PTGS2. Compared with normal bladder tissue, the level of PTGS2 in the bladder tissue of patients with cystitis or bladder cancer is increased [40]. PTGS2 is usually an indicator of poor prognosis for patients [41]. Cekanova et al. [42] suggested that the overexpression of PTGS2 could be a target for detection and treatment of bladder cancer. From our data, the mRNA expression of PPARG and PTGS2 were upregulated in two bladder cancer cell lines in comparison to the normal cells SV-HUC-1 (Fig. 5B). Importantly, SZY-200, CP-4126 and GEM could downregulate the expressions of the two genes in bladder cancer cells (Fig. 5C). As shown in Fig. S4 and S5, according to the KEGG biological pathway analyses, we found that these two genes (black arrows) are located downstream of the target genes of GEM. That's why these drugs could reduce the expression of these two genes.

\section{Conclusion}

In summary, these results indicate that SZY-200 has broad-spectrum anti-cancer activity, and the inhibitory effects on the proliferation of bladder cancer cells are comparable to GEM and CP-4126. The decrease in cell viability could be attributable to both cell-cycle arrest and apoptotic cell death. Additionally, similar to CP-4126, SZY-200 is expected to overcome GEM resistance induced by low hENT1 expression. Overall, SZY-200 has potential clinical application value. Further studies are required to determine whether SZY-200 has a favorable anti-tumor activity in vivo compared with GEM and CP-4126.

\section{Material And Method 4.1. Chemicals}

GEM were bought from Shanghai Makclin Biochemical Company. CP-4126 and SZY-200 were synthesized by Shao Zhiyu (Donghua University, Shanghai, China). They were dissolved in DMSO (Solarbio Life Science, Beijing, China) to a final concentration of $8 \mathrm{mM}$ and stored at $4{ }^{\circ} \mathrm{C}$ for in vitro assay. LA and EA were purchased from Aladdin (Shanghai, China).and Alfa Aesar (Ward Hill, MA), respectively. They were dissolved in absolute ethanol to a final concentration of $60 \mathrm{mM}$ and stored at room temperature. Nitrobenzylthioinosine (NBMPR) was purchased from Topscience (Shanghai, China) and dissolved in DMSO to a final concentration of $100 \mathrm{mM}$ and stored at $-20^{\circ} \mathrm{C}$. Propidium iodide (PI) was bought from Sangon Biotech (Shanghai, China). RNase A was purchased from Solarbio Life Science (Beijing, China).

\subsection{Cell culture}

The human normal uroepithelium cell line SV-HUC-1 was purchased from the American Type Culture Collection (ATCC; Manassas, VA, USA) and maintained in DMEM/F12 medium (Gibco/Invitrogen, Camarillo, CA, USA). Human bladder cancer cell lines (T24, UM-UC-3, 5637), human normal hepatocyte cell line QSG-7701, human lung cancer cell line A549 and colon cancer cell line HCT116 were acquired from the Chinese Academy of Sciences (Shanghai, China) and maintained in RPMI-1640 medium (Gibco) 
or DMEM (Gibco) and supplemented with $1 \%$ penicillin $\mathrm{G}$ sodium/streptomycin sulphate and $10 \%$ foetal bovine serum (FBS, PAN-Biotech, Aidenbach, Germany) in a humidified atmosphere composed of $5 \% \mathrm{CO}_{2}$ and $95 \%$ air at $37{ }^{\circ} \mathrm{C}$.

\subsection{Cell viability assay}

The cells in logarithmic phase were seeded in 96 -well plates and incubated at $37^{\circ} \mathrm{C}$ to allow cell attachment. Then treated with GEM, CP-4126 or SZY-200 (0, 1, 5, 25 and 50 nM), LA or EA (150 and 300 $\mu \mathrm{M})$ for $48 \mathrm{~h}$. The nucleoside transport carrier inhibitor NBMPR $(100 \mu \mathrm{M})$ was added into the cells for $1 \mathrm{~h}$ and removed before adding the drugs. The CCK-8 kit was used to measure cell viability according to the manufacturer's instructions (APExBIO, Houston, USA). In brief, after adding $10 \mu \mathrm{L} \mathrm{CCK-8} \mathrm{to} \mathrm{each} \mathrm{well} \mathrm{and}$ incubating for $1 \mathrm{~h}$ at $37^{\circ} \mathrm{C}$, absorbance at $450 \mathrm{~nm}$ was measured by the microplate reader (Bio-Rad, Hercules, USA). The software GraphPad Prism 8.4 (GraphPad Software, Inc.) was used to perform statistical analysis of cell viability results.

\subsection{Cell colony forming assay}

UM-UC-3 cells were seeded in 6-well plates (1000 cells $/ 2 \mathrm{~mL}$ medium/well), and incubated at $37^{\circ} \mathrm{C}$ for 24 $h$, then treated with LA and EA (150 and $300 \mu \mathrm{M})$ for 7-10 days for colony growth. After medium was removed, the cells were fixed with $4 \%$ paraformaldehyde for $20 \mathrm{~min}$ at room temperature, and stained with $0.1 \%$ crystal violet for $30 \mathrm{~min}$, and the plate was imaged and colonies were counted.

\subsection{Quantitative real-time PCR (qRT-PCR)}

Total RNA in cells was extracted using the Trizol reagent (Yeasen Biotech, Shanghai, China) and reversetranscribed using the Hifair ${ }^{\circledR} \otimes 1$ st Strand cDNA Synthesis kit (Yeasen Biotech) according to the manufacturer's instructions. qRT-PCR was performed on the C1000 Thermal Cycle system (Bio-Rad, Hercules, USA) using Hieff UNICON® qPCR SYBR Green Master Mix (Yeasen Biotech). Transcript quantities were compared by the relative $\mathrm{Ct}$ method. The value in relation to the control sample was given by $2^{-\triangle \Delta C T}$.

\subsection{Cell cycle analysis}

After incubated with drugs $(12.5 \mathrm{nM})$ in 6-well plates for $24 \mathrm{~h}$, the cells were harvested, centrifuged, and washed with cold PBS twice. Then fixed in $1 \mathrm{~mL}$ ice cold ethanol $(70 \%)$ at $-20^{\circ} \mathrm{C}$ overnight. After that, the cells were washed again with cold PBS and resuspended in cold PI solution $(50 \mu \mathrm{g} / \mathrm{ml})$ containing RNase $\mathrm{A}(0.1 \mathrm{mg} / \mathrm{ml})$ in PBS $(\mathrm{pH} 7.4)$ for 30 minutes at $37^{\circ} \mathrm{C}$ in the dark. Cell cycle was measured by a CytoFLEX flow cytometer (Beckman Coulter, Brea, USA).

\subsection{Apoptosis analysis}

The apoptosis of cells was observed using the Hoechst 33258 assay kit (Dojindo Laboratories, Tokyo, Japan). The cells were seeded in 6-well plates and treated with drugs (12.5 and $25 \mathrm{nM})$. After $48 \mathrm{~h}$, the attached cells were washed with PBS and fixed in freshly prepared 4\% paraformaldehyde for $20 \mathrm{~min}$ at room temperature, then washed with PBS and incubated with Hoechst 33258 staining solution for 10 min 
at $37^{\circ} \mathrm{C}$ in the dark. After that, cells were washed with PBS and added Antifade Mounting Medium, then observed under a fluorescence microscope (Olympus BX51, Tokyo, Japan).

The proportion of apoptotic cells was quantified using the Annexin V-FITC/PI Kit by flow cytometry. After incubation with drugs $(25 \mathrm{nM})$ in 6-well plates for $48 \mathrm{~h}$, the cells were harvested and centrifuged, washed with PBS, stained with $5 \mu \mathrm{L}$ Annexin V-FITC and $10 \mu \mathrm{L}$ propidium iodide in the dark at room temperature for 15 min according to the manufacturer's protocol (Yeasen Biotech) and then determined with a CytoFLEX flow cytometer (Beckman Coulter, Brea, USA). Data analysis were performed with FlowJo software.

\subsection{Western blot analysis}

The cells were seeded in 6-well plates and treated with different drugs (12.5 and $25 \mathrm{nM}$ ). After 24 or $48 \mathrm{~h}$, the cells were washed with ice-cold PBS and lysed on ice for $30 \mathrm{~min}$ in lysis buffer containing $400 \mathrm{mM}$ $\mathrm{NaCl}, 1.5 \mathrm{mM} \mathrm{MgCl}_{2}, 25 \mathrm{mM}$ HEPES (pH 7.7), 0.5\% Triton X-100, 2 mM EDTA, 10 mM DTT, 0.1 mM PMSF, $20 \mathrm{mM} \beta-\mathrm{GP}, 1 \mu \mathrm{M} \mathrm{Na} \mathrm{VO}_{4}$. The lysate was centrifuged at $12,000 \times \mathrm{g}$ for $15 \mathrm{~min}$, the supernatant was collected, and protein concentration was determined by Bradford assay. $40 \mu \mathrm{g}$ proteins from each sample were subjected to electrophoresis on $12 \%$ SDS-PAGE. The separated proteins were transferred onto a PVDF membrane. After blocked with 5\% skimmed milk in PBST at room temperature for $2 \mathrm{~h}$, the PVDF membrane was incubated with primary antibodies for GAPDH (KC-5G5, Aksomics, Shanghai, China), Bax (AF0120, Affinity biosciences OH, USA) and Bcl-2 (AF6139, Affinity biosciences OH, USA) at $4^{\circ} \mathrm{C}$ overnight, respectively. The membranes were washed three times (10 min per wash) and then incubated with a goat anti-rabbit IgG HRP secondary antibody (111-036-003, Jackson ImmunoResearch Laboratories, USA) at room temperature for approximately $2 \mathrm{~h}$ and washed again. The specific protein signals were visualized using ECL Protein Imprint Detection Kit (EpiZyme, Shanghai, China), followed by exposure with the Tanon 6200 Imaging System (Tanon Science \& Technology Co., Ltd, Shanghai, China).

\subsection{Wound healing assay}

The cells were seeded in 6-well plates and cultured until 100\% confluence. A scratch was created using a $10 \mu \mathrm{L}$ pipette tip and cells were washed with PBS. Serum-free medium containing different drugs was added to allow cells moving into the gap. At $0,20,28,40 \mathrm{~h}$, wound closure images were captured in the pre-marked same field under magnification. Cell motility was calculated as the ratio of the difference between the initial scratch area and the final scratch area of each sample to the initial scratch area.

\subsection{Explore target genes of GEM and LA}

The target genes of GEM and LA were predicted using BATMAN-TCM database (http://bionet.ncpsb.org.cn/batman-tcm/index.php).

\subsection{Statistical analysis}

All experiments were performed in triplicate and repeated at least three times. The data were expressed as the mean \pm SD using GraphPad Prism 8.4 statistical software. Statistical analysis was performed using 
Student's t-test. The $P$ value less than 0.05 was considered as statistically significant.

\section{Declarations}

Acknowledgements This work was supported by the National Key Research and Development Project of China [2018YFA0902804].

\section{Compliance with ethical standards}

Conflict of interest The authors declare no competing interests.

Publisher's note Springer Nature remains neutral with regard to jurisdictional claims in published maps and institutional affiliations.

\section{References}

1. Sung H, Ferlay J, Siegel RL, Laversanne M, Soerjomataram I, Jemal A et al (2021) Global Cancer Statistics 2020: GLOBOCAN Estimates of Incidence and Mortality Worldwide for 36 Cancers in 185 Countries. CA Cancer J Clin 71(3):209-249. doi:10.3322/caac.21660

2. Hurle R, Contieri R, Casale P, Morenghi E, Saita A, Buffi N et al (2021) Midterm follow-up (3 years) confirms and extends short-term results of intravesical gemcitabine as bladder-preserving treatment for non-muscle-invasive bladder cancer after BCG failure. Urol Oncol 39(3):195.e7-195.e.e13. doi:10.1016/j.urolonc.2020.09.017

3. Lu JL, Xia QD, Lu YH, Liu Z, Zhou P, Hu HL et al (2020) Efficacy of intravesical therapies on the prevention of recurrence and progression of non-muscle-invasive bladder cancer: A systematic review and network meta-analysis. Cancer Med 9(21):7800-7809. doi:10.1002/cam4.3513

4. von der Maase H, Hansen SW, Roberts JT, Dogliotti L, Oliver T, Moore MJ et al (2000) Gemcitabine and cisplatin versus methotrexate, vinblastine, doxorubicin, and cisplatin in advanced or metastatic bladder cancer: results of a large, randomized, multinational, multicenter, phase III study. J Clin Oncol 18(17):3068-3077. doi:10.1200/jco.2000.18.17.3068

5. Pfister C, Gravis G, Fléchon A, Soulié M, Guy L, Laguerre B et al (2021) Randomized Phase III Trial of Dose-dense Methotrexate, Vinblastine, Doxorubicin, and Cisplatin, or Gemcitabine and Cisplatin as Perioperative Chemotherapy for Patients with Muscle-invasive Bladder Cancer. Analysis of the GETUG/AFU V05 VESPER Trial Secondary Endpoints: Chemotherapy Toxicity and Pathological Responses. Eur Urol 79(2):214-221. doi:10.1016/j.eururo.2020.08.024

6. Marcé S, Molina-Arcas M, Villamor N, Casado FJ, Colomer D (2006) Expression of human equilibrative nucleoside transporter 1 (hENT1) and its correlation with gemcitabine uptake and cytotoxicity in mantle cell lymphoma. Haematologica 91(7):895-902

7. Aoyama T, Kazama K, Miyagi Y, Murakawa M, Yamaoku K, Atsumi Y et al (2017) Predictive role of human equilibrative nucleoside transporter 1 in patients with pancreatic cancer treated by curative 
resection and gemcitabine-only adjuvant chemotherapy. Oncol Lett 14(1):599-606. doi:10.3892/ol.2017.6220

8. Bird NT, Elmasry M, Jones R, Psarelli E, Dodd J, Malik H et al (2017) Immunohistochemical hENT1 expression as a prognostic biomarker in patients with resected pancreatic ductal adenocarcinoma undergoing adjuvant gemcitabine-based chemotherapy. Br J Surg 104(4):328-336. doi:10.1002/bjs.10482

9. Farrell JJ, Elsaleh H, Garcia M, Lai R, Ammar A, Regine WF et al (2009) Human equilibrative nucleoside transporter 1 levels predict response to gemcitabine in patients with pancreatic cancer. Gastroenterology 136(1):187-195. doi:10.1053/j.gastro.2008.09.067

10. Maréchal R, Mackey JR, Lai R, Demetter P, Peeters M, Polus M et al (2009) Human equilibrative nucleoside transporter 1 and human concentrative nucleoside transporter 3 predict survival after adjuvant gemcitabine therapy in resected pancreatic adenocarcinoma. Clin Cancer Res 15(8):29132919. doi:10.1158/1078-0432.ccr-08-2080

11. Kim R, Tan A, Lai KK, Jiang J, Wang Y, Rybicki LA et al (2011) Prognostic roles of human equilibrative transporter 1 (hENT-1) and ribonucleoside reductase subunit M1 (RRM1) in resected pancreatic cancer. Cancer 117(14):3126-3134. doi:10.1002/cncr.25883

12. Liu ZQ, Han YC, Zhang X, Chu L, Fang JM, Zhao HX et al (2014) Prognostic value of human equilibrative nucleoside transporter1 in pancreatic cancer receiving gemcitabin-based chemotherapy: a meta-analysis. PloS one 9(1):e87103. doi:10.1371/journal.pone.0087103

13. Bergman AM, Pinedo HM, Peters GJ. Determinants of resistance to 2',2'-difluorodeoxycytidine (gemcitabine). Drug resistance updates: reviews and commentaries in antimicrobial and anticancer chemotherapy. 2002;5(1):19-33. doi:10.1016/s1368-7646(02)00002-x

14. Heinemann V, Hertel LW, Grindey GB, Plunkett W (1988) Comparison of the cellular pharmacokinetics and toxicity of 2',2'-difluorodeoxycytidine and 1-beta-D-arabinofuranosylcytosine. Cancer Res 48(14):4024-4031

15. Veltkamp SA, Jansen RS, Callies S, Pluim D, Visseren-Grul CM, Rosing $\mathrm{H}$ et al (2008) Oral administration of gemcitabine in patients with refractory tumors: a clinical and pharmacologic study. Clin Cancer Res 14(11):3477-3486. doi:10.1158/1078-0432.ccr-07-4521

16. Bergman AM, Kuiper CM, Noordhuis P, Smid K, Voorn DA, Comijn EM et al (2004) Antiproliferative activity and mechanism of action of fatty acid derivatives of gemcitabine in leukemia and solid tumor cell lines and in human xenografts. Nucleosides Nucleotides Nucleic Acids 23(8-9):13291333. doi:10.1081/NCN-200027579

17. Bergman AM, Adema AD, Balzarini J, Bruheim S, Fichtner I, Noordhuis P et al (2011) Antiproliferative activity, mechanism of action and oral antitumor activity of CP-4126, a fatty acid derivative of gemcitabine, in in vitro and in vivo tumor models. Invest New Drugs 29(3):456-466. doi:10.1007/s10637-009-9377-7

18. Galmarini CM, Myhren F, Sandvold ML (2009) CP-4055 and CP-4126 are active in ara-C and gemcitabine-resistant lymphoma cell lines. Br J Haematol 144(2):273-275. doi:10.1111/j.1365- 
19. Adema AD, Smid K, Losekoot N, Honeywell RJ, Verheul HM, Myhren F et al (2012) Metabolism and accumulation of the lipophilic deoxynucleoside analogs elacytarabine and CP-4126. Invest New Drugs 30(5):1908-1916. doi:10.1007/s10637-011-9756-8

20. Tanabe E, Kitayoshi M, Fujii K, Ohmori H, Luo Y, Kadochi Y et al (2017) Fatty acids inhibit anticancer effects of 5-fluorouracil in mouse cancer cell lines. Oncol Lett 14(1):681-686. doi:10.3892/ol.2017.6190

21. DeLany JP, Windhauser MM, Champagne CM, Bray GA (2000) Differential oxidation of individual dietary fatty acids in humans. Am J Clin Nutr 72(4):905-911. doi:10.1093/ajcn/72.4.905

22. Soliman S, Faris ME, Ratemi Z, Halwani R (2020) Switching Host Metabolism as an Approach to Dampen SARS-CoV-2 Infection. Ann Nutr Metab 76(5):297-303. doi:10.1159/000510508

23. Nonaka Y, Takagi T, Inai M, Nishimura S, Urashima S, Honda K et al (2016) Lauric Acid Stimulates Ketone Body Production in the KT-5 Astrocyte Cell Line. J Oleo Sci 65(8):693-699. doi:10.5650/jos.ess16069

24. Lappano R, Sebastiani A, Cirillo F, Rigiracciolo DC, Galli GR, Curcio R et al (2017) The lauric acidactivated signaling prompts apoptosis in cancer cells. Cell Death Discov 3:17063. doi:10.1038/cddiscovery.2017.63

25. Venugopal B, Awada A, Evans TR, Dueland S, Hendlisz A, Rasch W et al (2015) A first-in-human phase I and pharmacokinetic study of CP-4126 (CO-101), a nucleoside analogue, in patients with advanced solid tumours. Cancer Chemother Pharmacol 76(4):785-792. doi:10.1007/s00280-0152846-0

26. Bergman AM, Kuiper CM, Noordhuis P, Smid K, Voorn DA, Comijn EM et al (2004) Antiproliferative activity and mechanism of action of fatty acid derivatives of gemcitabine in leukemia and solid tumor cell lines and in human xenografts. Nucleosides Nucleotides Nucleic Acids 23(8-9):13291333. doi:10.1081/ncn-200027579

27. Liu R, Jiang Y, Hu X, Wu J, Jiang W, Jin G et al (2018) A preclinical evaluation of cytarabine prodrug nanofibers assembled from cytarabine-lauric acid conjugate toward solid tumors. Int J Pharm 552(1-2):111-118. doi:10.1016/j.ijpharm.2018.09.043

28. Achiwa H, Oguri T, Sato S, Maeda H, Niimi T, Ueda R (2004) Determinants of sensitivity and resistance to gemcitabine: the roles of human equilibrative nucleoside transporter 1 and deoxycytidine kinase in non-small cell lung cancer. Cancer Sci 95(9):753-757. doi:10.1111/j.13497006.2004.tb03257.x

29. Mori R, Ishikawa T, Ichikawa Y, Taniguchi K, Matsuyama R, Ueda M et al (2007) Human equilibrative nucleoside transporter 1 is associated with the chemosensitivity of gemcitabine in human pancreatic adenocarcinoma and biliary tract carcinoma cells. Oncol Rep 17(5):1201-1205

30. Neoptolemos JP, Greenhalf W, Ghaneh P, Palmer DH, Cox TF, Garner E et al. HENT1 tumor levels to predict survival of pancreatic ductal adenocarcinoma patients who received adjuvant gemcitabine and adjuvant 5FU on the ESPAC trials. Journal of Clinical Oncology. 2013;31(15) 
31. Wang X, Bai Y, Zhang F, Yang Y, Feng D, Li A et al (2020) Targeted Inhibition of P4HB Promotes Cell Sensitivity to Gemcitabine in Urothelial Carcinoma of the Bladder. Onco Targets Ther 13:9543-9558. doi:10.2147/OTT.S267734

32. Yang Y, Zhang LJ, Bai XG, Xu HJ, Jin ZL, Ding M (2018) Synergistic antitumour effects of triptolide plus gemcitabine in bladder cancer. Biomedicine pharmacotherapy = Biomedecine pharmacotherapie 106:1307-1316. doi:10.1016/j.biopha.2018.07.083

33. Smith MR, Joshi I, Jin F, Obasaju C (2005) Enhanced efficacy of gemcitabine in combination with anti-CD20 monoclonal antibody against CD20 + non-Hodgkin's lymphoma cell lines in vitro and in scid mice. BMC Cancer 5:103. doi:10.1186/1471-2407-5-103

34. da Silva GN, de Castro Marcondes JP, de Camargo EA, da Silva Passos Junior GA, Sakamoto-Hojo ET, Salvadori DM (2010) Cell cycle arrest and apoptosis in TP53 subtypes of bladder carcinoma cell lines treated with cisplatin and gemcitabine. Exp Biol Med (Maywood) 235(7):814-824. doi:10.1258/ebm.2010.009322

35. Pinto-Leite R, Arantes-Rodrigues R, Palmeira C, Gaivao I, Cardoso ML, Colaco A et al (2012) Everolimus enhances gemcitabine-induced cytotoxicity in bladder-cancer cell lines. J Toxicol Environ Health A 75(13-15):788-799. doi:10.1080/15287394.2012.690325

36. Ogino S, Shima K, Baba Y, Nosho K, Irahara N, Kure S et al (2009) Colorectal cancer expression of peroxisome proliferator-activated receptor gamma (PPARG, PPARgamma) is associated with good prognosis. Gastroenterology 136(4):1242-1250. doi:10.1053/j.gastro.2008.12.048

37. Goldstein JT, Berger AC, Shih J, Duke FF, Furst L, Kwiatkowski DJ et al (2017) Genomic Activation of PPARG Reveals a Candidate Therapeutic Axis in Bladder Cancer. Cancer Res 77(24):6987-6998. doi:10.1158/0008-5472.can-17-1701

38. Yang DR, Lin SJ, Ding XF, Miyamoto H, Messing E, Li LQ et al (2013) Higher expression of peroxisome proliferator-activated receptor $\mathrm{y}$ or its activation by agonist thiazolidinedionerosiglitazone promotes bladder cancer cell migration and invasion. Urology 81(5):1109.e1-1109.e6. doi:10.1016/j.urology.2012.12.027

39. Cheng S, Qian K, Wang Y, Wang G, Liu X, Xiao Y et al (2019) PPARY inhibition regulates the cell cycle, proliferation and motility of bladder cancer cells. J Cell Mol Med 23(5):3724-3736. doi: $10.1111 / \mathrm{jcmm} .14280$

40. Wheeler MA, Hausladen DA, Yoon JH, Weiss RM (2002) Prostaglandin E2 production and cyclooxygenase-2 induction in human urinary tract infections and bladder cancer. The Journal of urology 168(4 Pt 1):1568-1573. doi:10.1097/01.ju.0000030583.31299.80

41. Kömhoff M, Guan Y, Shappell HW, Davis L, Jack G, Shyr Y et al (2000) Enhanced expression of cyclooxygenase-2 in high grade human transitional cell bladder carcinomas. Am J Pathol 157(1):29-35. doi:10.1016/s0002-9440(10)64513-0

42. Cekanova M, Uddin MJ, Bartges JW, Callens A, Legendre AM, Rathore K et al (2013) Molecular imaging of cyclooxygenase-2 in canine transitional cell carcinomas in vitro and in vivo. Cancer prevention research (Philadelphia Pa) 6(5):466-476. doi:10.1158/1940-6207.capr-12-0358 
Figures

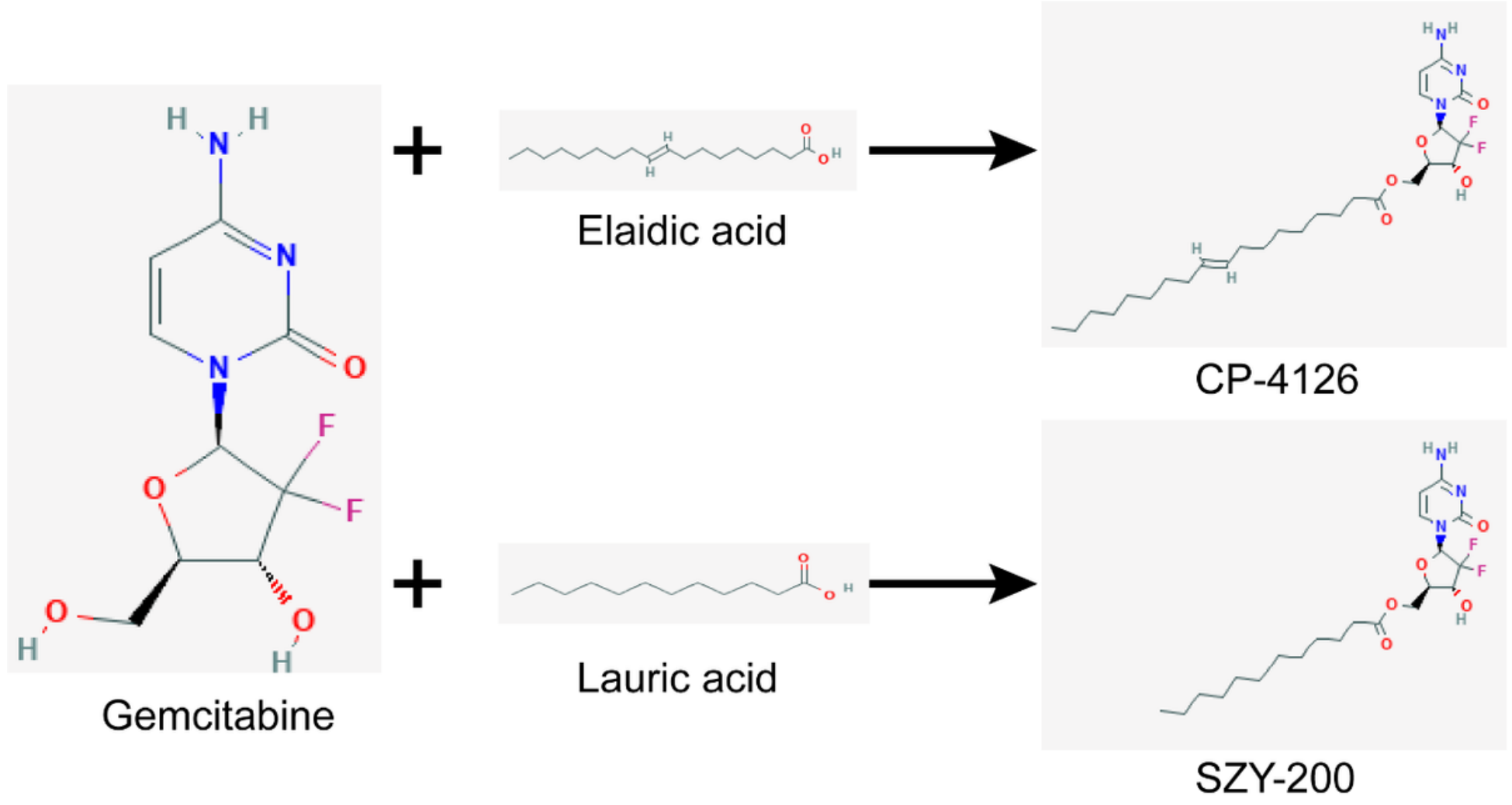

Figure 1

Design strategies and chemical structures of GEM derivatives CP-4126 and SZY-200 
(A)
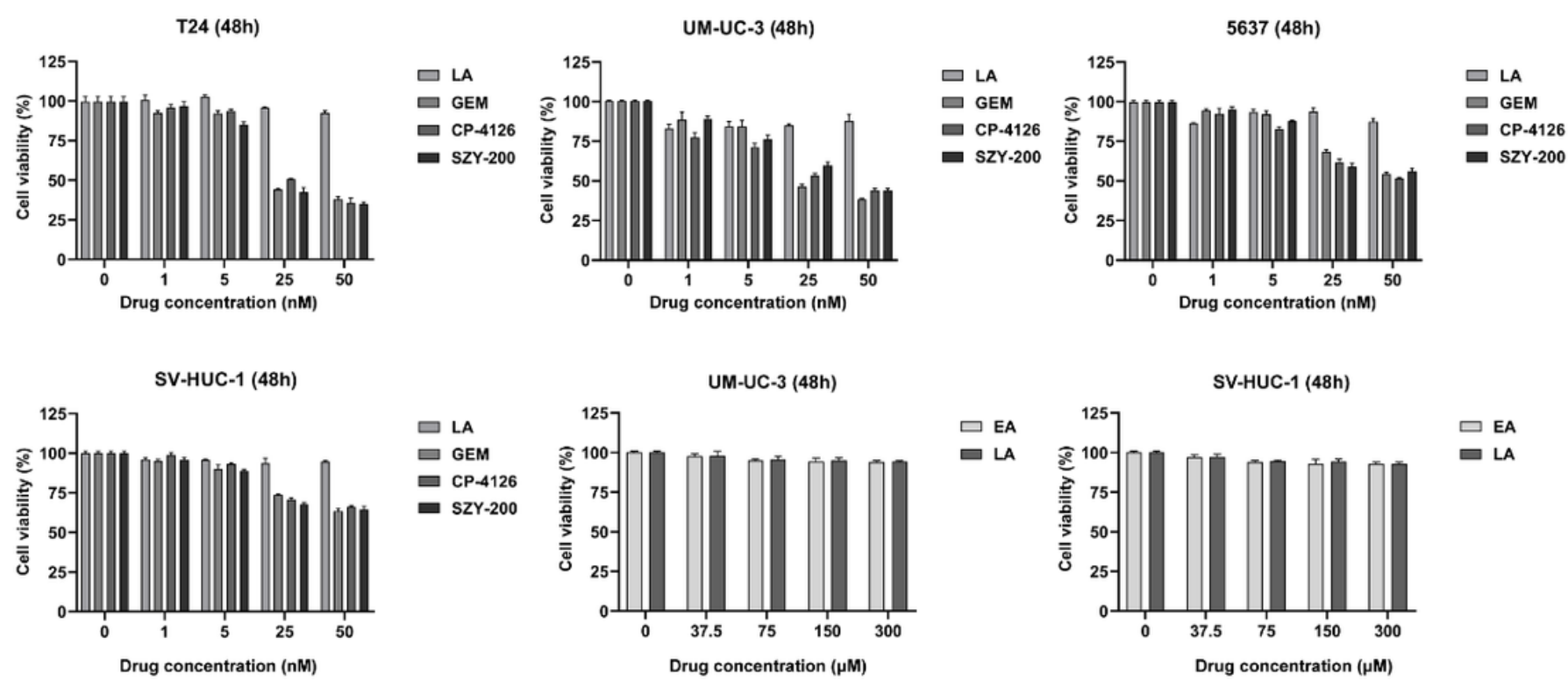

(B)

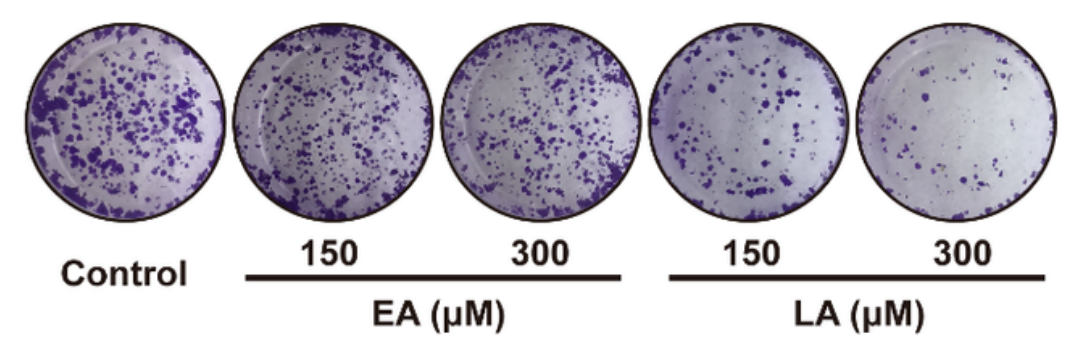

(C)

UM-UC-3

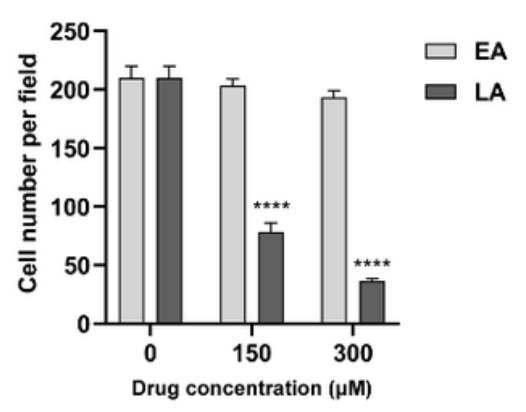

Figure 2

Antiproliferative activities of SZY-200 and LA against tumor cell lines with CP-4126, GEM and EA as positive control. (A) Cell viability after treatment for $48 \mathrm{~h}$. (B) Colony formation abilities of UM-UC-3 cells was determined after treatment with EA or LA. (C) Statistical analysis of (B). Values are expressed as the mean \pm SD from three independent experiments. ${ }^{\star \star \star \star} p<0.0001$ vs control. Abbreviations: GEM, GEM; EA, elaidic acid; LA, lauric acid 
(A)

hENT1

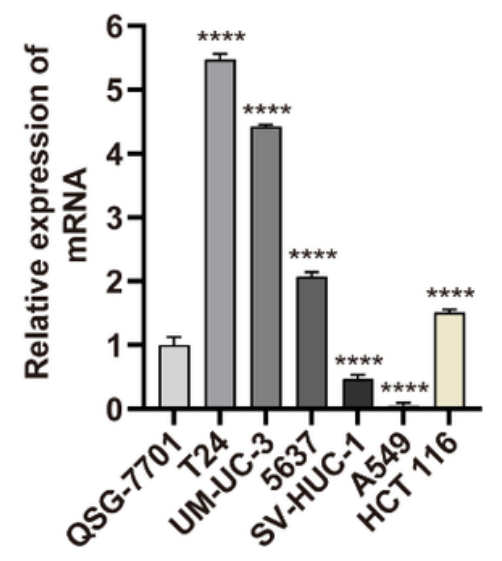

(C)

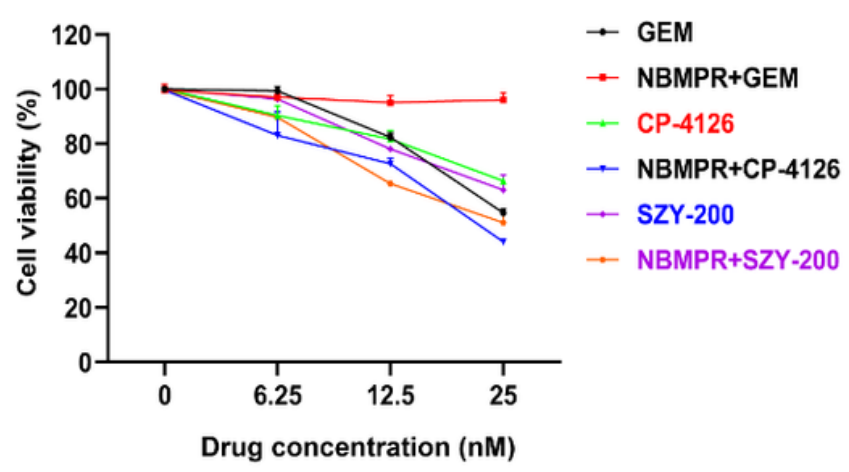

(B)

T24(48h)

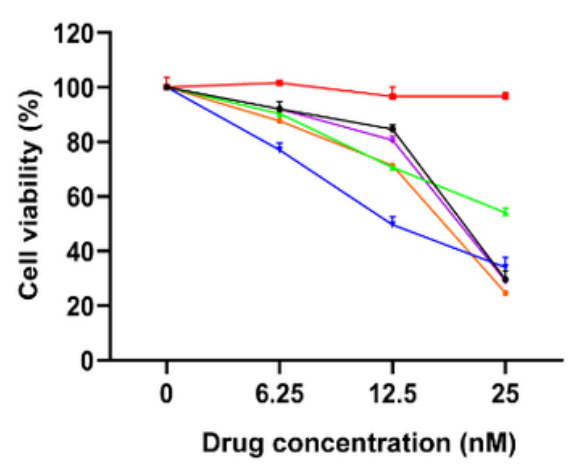

- GEM

$\rightarrow$ NBMPR+GEM

- CP-4126

- NBMPR+CP-4126

- SZY-200

- NBMPR+SZY-200

(D)

5637 (48h)

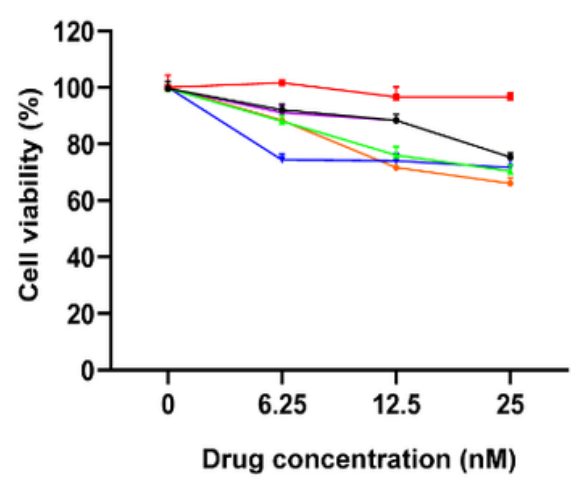

- GEM

$\rightarrow$ NBMPR+GEM

- CP-4126

- NBMPR+CP-4126

$\rightarrow$ SZY-200

- NBMPR+SZY-200

Figure 3

The expressions of hENT1 at the transcriptional level in different cells and the effects of the nucleoside transport inhibitor NBMPR on the cytotoxic activities of SZY-200, GEM and CP-4126 in bladder cancer cells. (A) The expression of hENT1 in different cells, $\star \star \star * P<0.0001$ vs. the normal cells QSG-7701. Cell viability was measured at $48 \mathrm{~h}$ after T24 cells (B), UM-UC-3 cells (C) and 5637 cells (D) were treated with SZY-200, GEM and CP-4126 $(6.25,12.5$ and $25 \mathrm{nM})$ with or without $100 \mu \mathrm{M}$ NBMPR. Data were mean \pm S.D. $(n=3)$ 
(A)

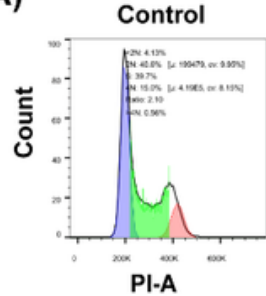

(C)

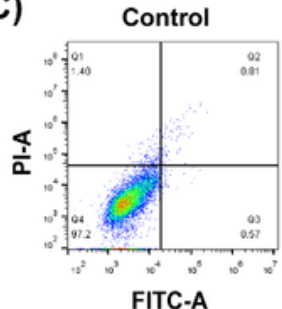

(E)

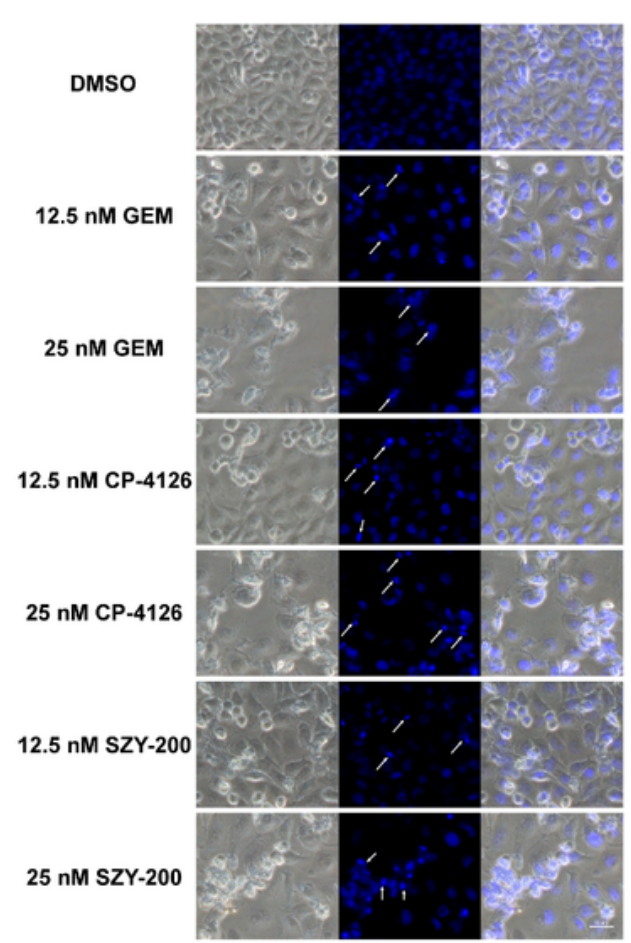

12.5 nM CP-4126
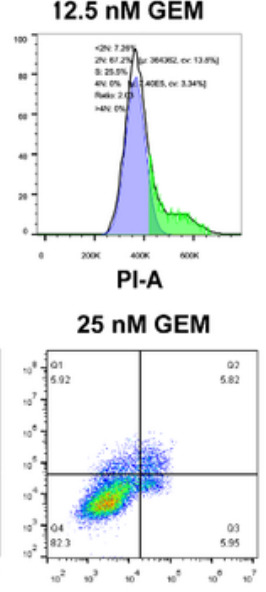

FITC-A

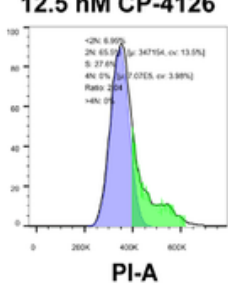

25 nM CP-4126

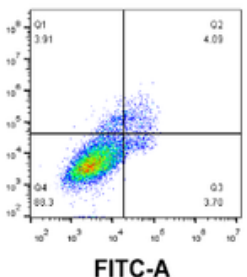

FITC-A
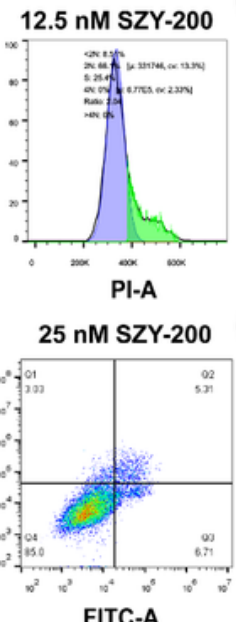

(B)

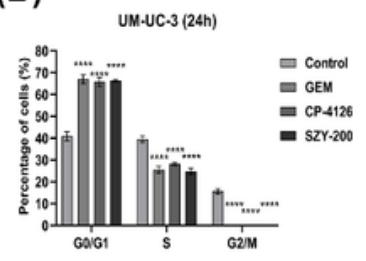

(D)

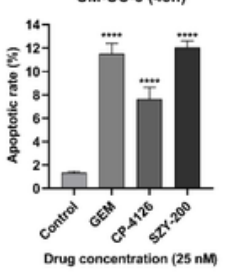

(F)

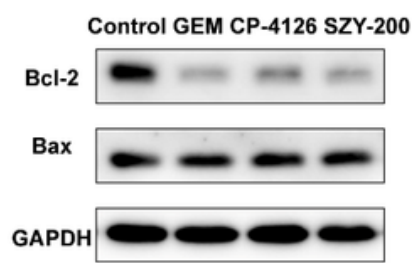

(G)

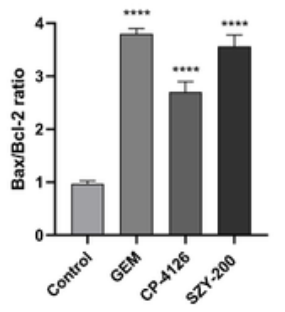

(I)

UM-UC-3 (48h)

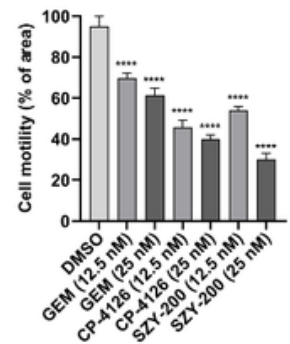

(H)
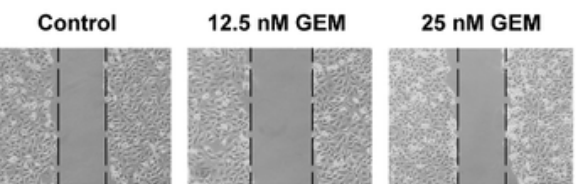

12.5 nM CP-4126
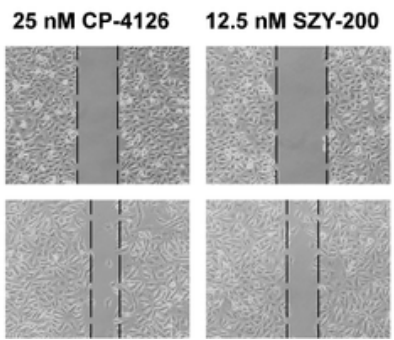

$25 \mathrm{nM} \mathrm{SZY-200}$
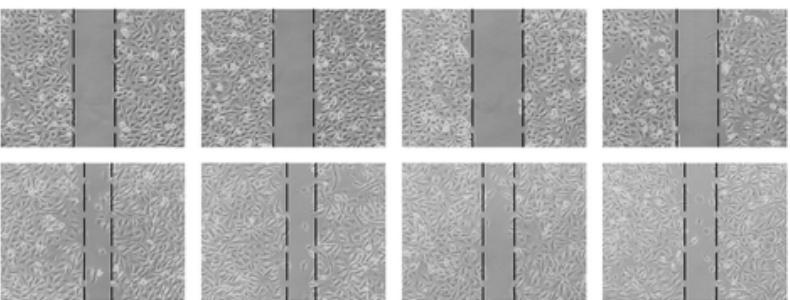

\section{Figure 4}

Effects of SZY-200 on cell cycle progression and the apoptosis rate in UM-UC-3 cells and scratch tests.

(A) After incubation, the cell cycle of UM-UC-3 cells was analyzed by flow cytometry. (B) Statistical analysis of the percentage of cells at the G0/G1, S and G2/M phases of cell cycle. (C) Apoptotic analysis was performed by flow cytometry using Annexin V-FITC/PI double staining method. (D) Statistical analysis of the percentages of apoptotic cells. (E) Apoptosis in UM-UC-3 cells was observed by 
fluorescence microscope using Hoechst 33258 staining (Scale bar $=50 \mu \mathrm{m})$. (F) Cell apoptosis-related protein expression in UM-UC-3 cells was detected by western blotting. (G) Represents the ratio of Bax/Bcl2 protein expression levels. ${ }^{\star} \star \star \star P \bigotimes 0.0001$ vs control. $(\mathrm{H})$ Representative wound-healing assay pictures for UM-UC-3 cells treated with DMSO or with three drugs (12.5 and $25 \mathrm{nM}$ ) are shown (vertical lines indicate wound edges); (400x, original magnification). (I) Histograms represent quantitative analyses of cell migration. Values are expressed as the mean \pm S.D. from three independent experiments. ${ }^{*} \star \star P \unrhd 0.0001$ vs DMSO

(A)

CMPK1(known target in DrugBank)

RRM1 (known target in DrugBank, KEGG)

TYMS (known target in DrugBank, TTD)

SHMT1, POLB, DNMT1, AK1, TBPL1, INSR, DPYD, HOXA5, FBLN1,

RRM2B, ADK, NT5M, DHODH, UCKL1, CMPK2, DPYS, DUT, RRM2,

AK9, CDKN1A, HIF 1A, MTOR, UMPS, AK7, HYAL2, CPS1, UCK1,

CAD, AK8, UPRT, DTYMK, UCK2, POLE, PPAT, POLE3, GART,

POLA1, DCK, FPGS, PNP, IMPDH1, ATIC, DHFR, POLE2, IMPDH2, POLE4, HPRT1
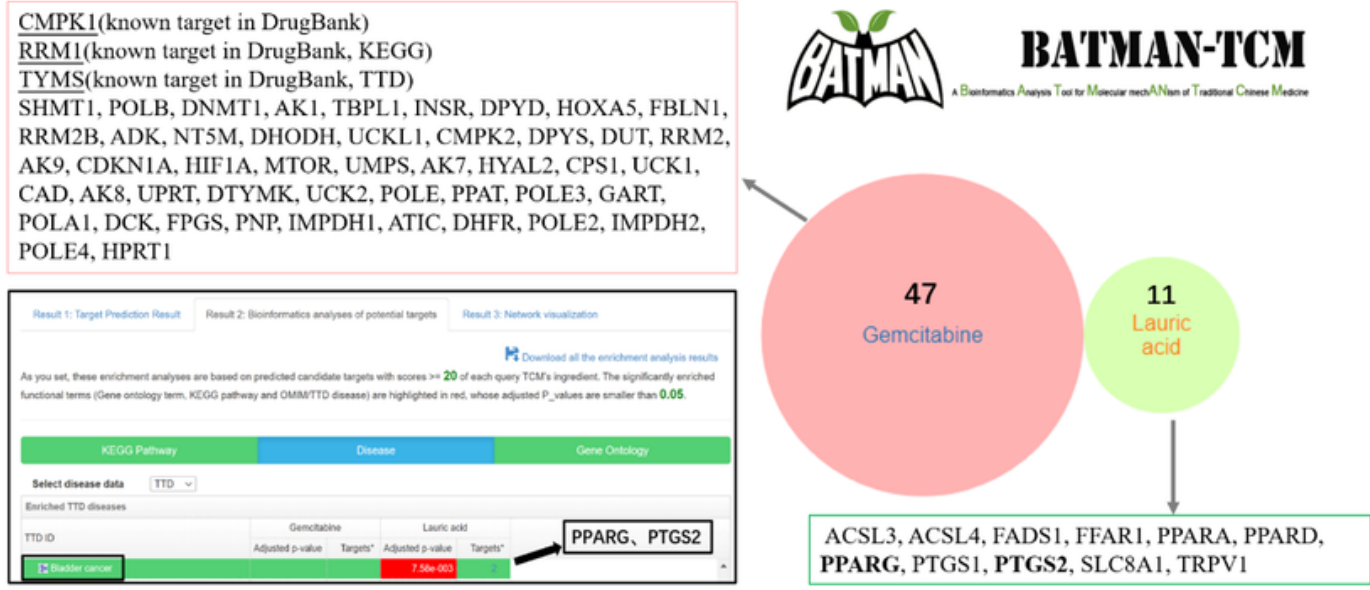

(B)
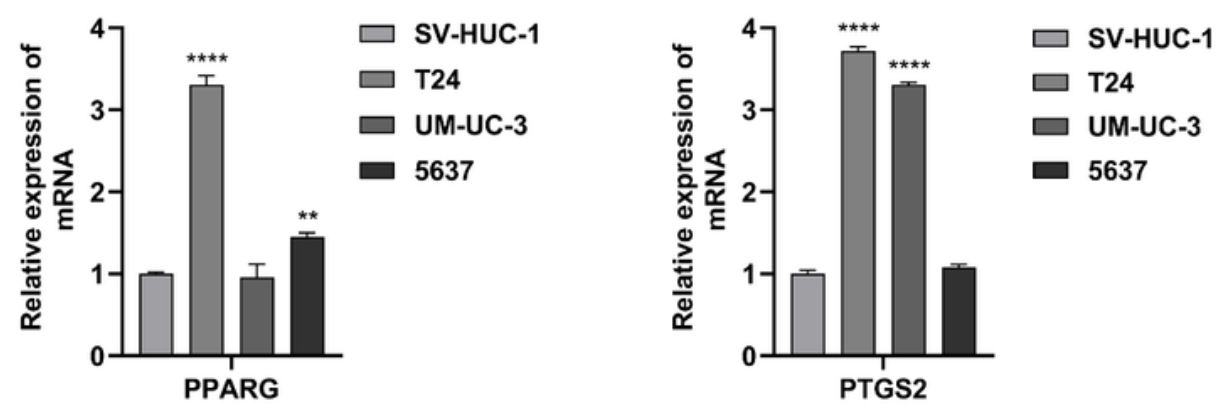

(C)
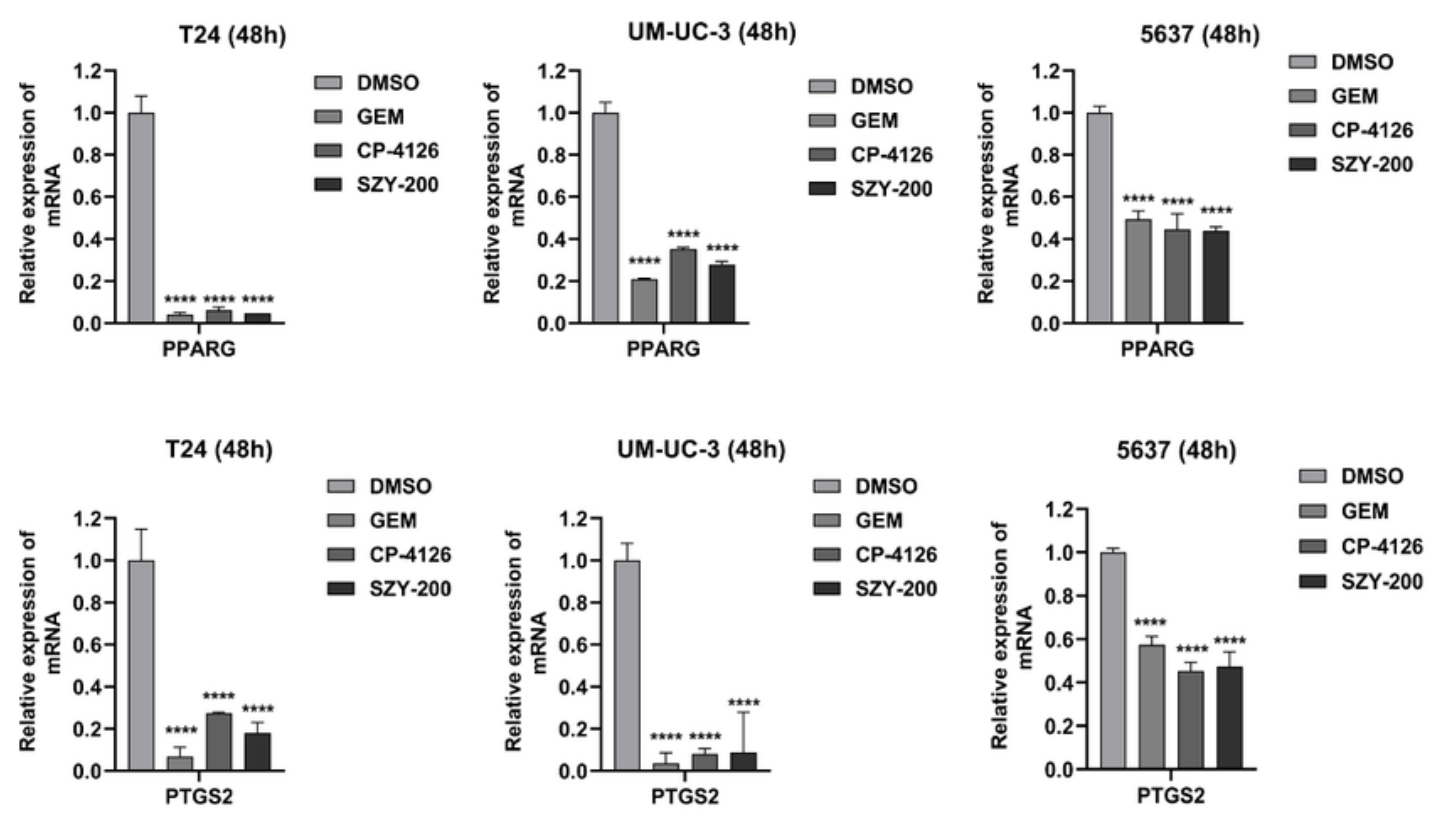


\section{Figure 5}

Prediction and analysis of target genes of GEM and LA. (A) Target genes from BATMAN-TCM database and disease enrichment analysis from TTD database. (B) Differential expressions of PPARG and PTGS2

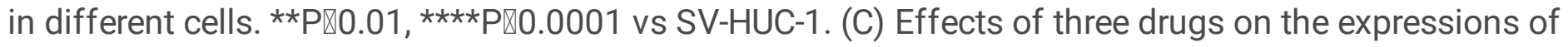
PPARG and PTGS2 in bladder cancer cells. Values are expressed as the mean \pm SD from three independent experiments. ${ }^{* \star \star * P} \otimes 0.0001$ vs DMSO

\section{Supplementary Files}

This is a list of supplementary files associated with this preprint. Click to download.

- Appendices.pptx 